\title{
Fetal Brain to Liver Weight Ratio as a Measure of Intrauterine Growth Retardation: Analysis of 182 Stillborn Autopsies
}

\author{
Mark L. Mitchell, M.D. \\ Department of Pathology, Christiana Care Health Services, Newark, Delaware
}

\begin{abstract}
The fetal brain/liver weight ratio of 182 stillborn fetuses was analyzed for its value as a measure of intrauterine growth retardation. The ratio was evaluated as a test for the detection of small-forgestational age fetuses, compared with the ponderal index, and evaluated for correlation with maternal histories that were compatible with fetal growth retardation. Both brain/liver ratio and ponderal index were insensitive and relatively nonspecific indicators of low fetal body weight in this population. Brain/liver weight ratio was considerably more sensitive in those cases that were at least 20 weeks of gestational age, and specificity increased as the cutoff point was increased above 3 . Those cases with maternal history or placental findings compatible with asymmetrical-type intrauterine growth retardation were statistically more likely to have elevated brain/liver weight ratios and depressed ponderal indices, but there was considerable overlap among cases with different disease types. Prosectors of fetal autopsies must make use of all the information available to them. The fetal brain/liver weight ratio and the ponderal index may be useful, but they are not satisfactory indicators of intrauterine growth retardation by themselves, and they also may be inadequate for the detection of growth retardation etiology.
\end{abstract}

KEY WORDS: Anthropometry, Fetal development, Fetal growth retardation, Intrauterine growth retardation, Pathology, Small for gestational age.

Mod Pathol 2001;14(1):14-19

Copyright $(\subset) 2001$ by The United States and Canadian Academy of Pathology, Inc.

VOL. 14, NO. 1, P. 14, 2001 Printed in the U.S.A.

Date of acceptance:

Address reprint requests to: Mark L. Mitchell, M.D., Department of Pathology and Laboratory Medicine, Christiana Hospital, Newark, DE 19718; e-mail: mmitchell@christianacare.org.
Newborn infants in the bottom 10th quantile for body weight are termed small for gestational age (SGA; 1). Some are constitutionally small, but others were unable to achieve their growth potential because of intrauterine growth retardation (IUGR; 2). Growth-retarded infants have increased perinatal morbidity and mortality $(1,3)$. Detection of the effects of IUGR is also important in the stillborn fetus at the time of autopsy because it may be a large contributor to understanding the etiology of their fatal outcome, and its presence may be helpful in guiding prenatal care in future pregnancies.

A common scheme for the classification of growth-retarded fetuses has been the classification into symmetrical (type I, proportionate, intrinsic, hypoplastic) and asymmetrical (type II, disproportionate, extrinsic, hypotrophic) forms $(1,4-6)$. Symmetrical growth retardation has been attributed to infection before 14 weeks gestation (e.g., $\mathrm{CMV}$, toxoplasmosis, rubella) and various malformation syndromes, especially Trisomies 13, 18, and 21. Fetuses with symmetrical growth retardation, according to theory, appear small but have normal body proportions. The chance that they might have later gestational growth, to compensate for early gestational failure to grow, is thought to be small. Asymmetrical growth retardation is usually due to placental insufficiency from maternal hypertension, multiple pregnancy, maternal malnutrition, or maternal smoking. Fetuses with asymmetrical growth appear disproportionate, with sparing of brain development at the expense of other somatic tissues $(1,3-5,7-9)$.

A number of different ultrasonographic indices have been used for the detection of the SGA fetus during development in utero. These have included the ratio of the head to abdominal circumference, the ratio of middle cerebral artery to umbilical artery blood flow, and measures of fetal thinness such as the ponderal index $(7,10-16)$. At the time of autopsy, the prosector may directly weigh the fetus and make appropriate measurements, but how is a constitutionally small fetus distinguished from one 
with IUGR? The ratio of the postmortem fetal brain to liver weight has been used for this purpose (5). Fetuses with asymmetrical growth retardation should, according to theory, show relative preservation of brain mass along with thin body habitus. The fetal brain is normally about three times the weight of the liver, but in asymmetrical-type IUGR, the value may increase to two or three times that value. The ratio is thought to be relatively independent of gestational age and theoretically provides a measure of IUGR independent of fetal body weight (5).

Data collected from 231 stillborn fetuses with postmortem examinations at Christiana Hospital were reviewed to analyze the characteristics of the brain/liver weight ratio. To attempt to measure the value of the ratio for the detection of IUGR, its relationship with low fetal body weight, with maternal history, and with ponderal index was investigated.

\section{METHODS}

There were 231 cases collected during a 6-year period. Of these, 49 cases were eliminated from the study because the brain/liver weight ratio could not be determined. Cases were placed in diagnostic classes based on review of the clinical abstract on the autopsy report and the placental findings (Table 1). Class 1 fetuses were thought to be likely to have asymmetrical growth retardation, Class 2 fetuses were thought to be at risk for symmetrical growth retardation, and Class 3 fetuses had no evidence to suggest risk for growth retardation. In addition to brain/liver weight ratio, the ponderal index was calculated (body weight in grams $\times 100$ divided by crown-to-heel length in centimeters cubed). The ponderal index had not been used at the time of autopsy but was calculated specifically for examination in this study. An additional three fetuses were eliminated from ponderal index calculations; a fetus with arthrogryposis had a small crown-heel length, and two fetuses had no measured crownheel length. In day-to-day use, the brain/liver ratio had been considered elevated if greater than 3 . The cutoff value for abnormal ponderal index is less certain; most studies have been of liveborn infants,

TABLE 1. Diagnostic Classes

\begin{tabular}{ll}
\hline Class & \multicolumn{1}{c}{ Typical Diagnoses } \\
\hline 1 & Placental insufficiency \\
& Maternal anemia \\
& Twin-twin transfusion syndrome \\
& Trisomy 18 \\
2 & Trisomy 21 \\
& Multiple congenital anomalies \\
& Acute chorioamnionitis \\
3 & Maternal cervical incompetence \\
\hline
\end{tabular}

and there is some evidence of age-related ponderal index variability late in gestation (16-18), so possible cutoff values were examined systematically for our population. Statistical analysis was performed using simple templates in a spreadsheet program and with the software application JMP (Macintosh Version 3.2.2; SAS, Cary, NC). Normal weight quantiles were measured using the data of GuihardCosta et al. (19) for 5000 singleton pregnancies in Paris, France without malformations or genetic disorders.

\section{RESULTS}

A scatter plot of the birth weight quantiles of 182 fetuses against their brain/liver weight ratios showed that of those fetuses with body weights at or below the 10th quantile, an almost equal number were above and below a brain/liver ratio of 3 (Fig. 1). Seven of the SGA fetuses were visible with high brain/liver weight ratio between 8 and 12, yielding a small second modal cluster. When these same data were examined by diagnostic class, there was a clear tendency for Class 1 fetuses to have higher brain/liver weight ratios $(P<0.0001$, ANOVA; $P<$ 0.05 , Hsu's MCB), but there was extensive overlap of diagnostic classes (Figs. 2, 3).

The brain/liver weight ratio was analyzed as a test for the detection of the SGA fetus. Table 2 shows the sensitivity and specificity of the test for each of five different cutoff points of brain/liver weight ratio. With a cutoff point of 3 , brain/liver

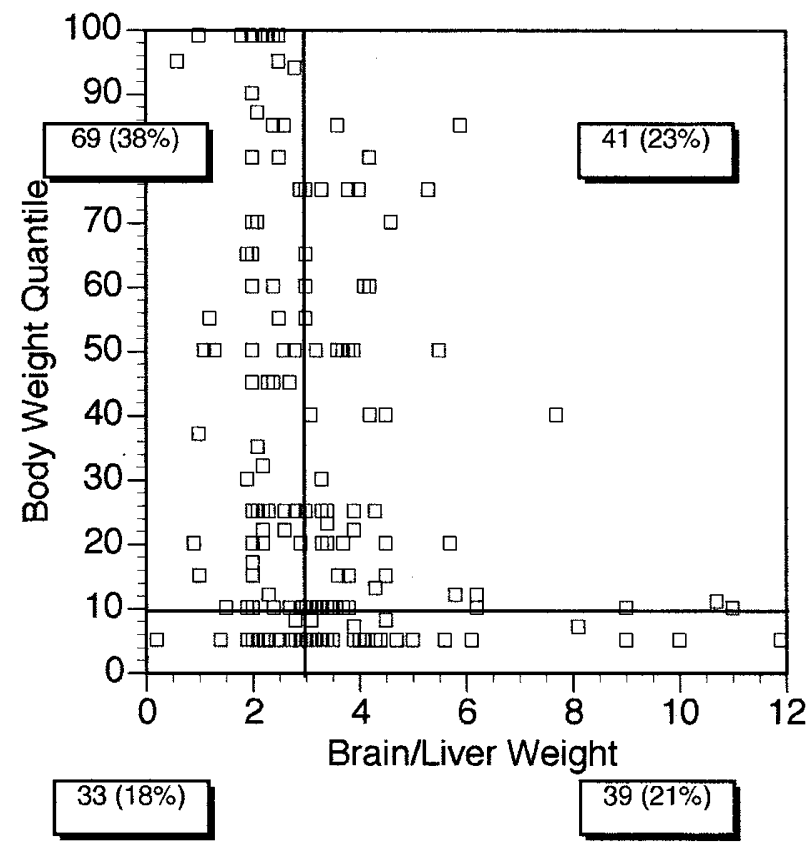

FIGURE 1. Fetal body weight quantile versus brain/liver weight ratio. Reference lines drawn at brain/liver weight ratio $=3$ and body weight quantile $=10$. Numbers and percentages in boxes are for the cases in each quadrant made by the reference lines. 


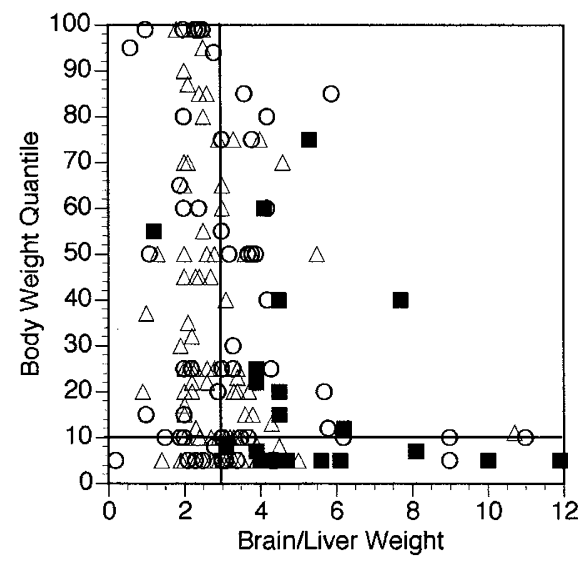

FIGURE 2. Fetal body weight quantile versus brain/liver weight ratio. Reference lines drawn at brain/liver weight ratio $=3$ and body weight quantile $=10$. Square data marker $=$ Class 1 disease, circular data marker $=$ Class 2 disease, triangular data marker $=$ Class 3 disease.

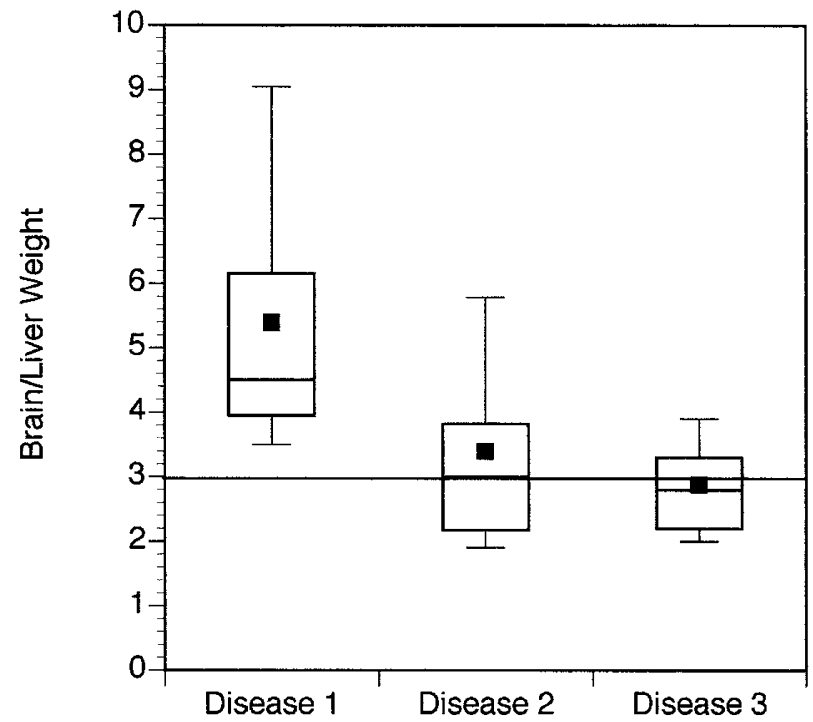

FIGURE 3. Box plots of each disease class distributed by brain/liver weight ratio. Disease Class 1 , likely associated with asymmetrical growth retardation, is different from Classes 2 and 3 by Hsu's MCB test at $\alpha=0.05$.

TABLE 2. Sensitivity and Specificity of Brain/Liver Weight Ratio for the Detection of SGA Fetus

\begin{tabular}{lrcrrr} 
B/L cutoff: & 3 & 3.7 & 4 & 5 & 6 \\
Sensitivity & 55 & 32 & 23 & 13 & 11 \\
Specificity & 63 & 77 & 84 & 93 & 97 \\
\hline
\end{tabular}

weight ratio was an insensitive and relatively nonspecific test for the detection of SGA. As the cutoff point increased, the test became more specific, but as expected, it became quite insensitive.

Some authors reserve the term small for gestational age for a fetus weighing well below the 10th quantile (1). Also, there is some evidence that asymmetrical-type IUGR is uncommon before 20 weeks of gestation $(5,20)$. Table 3 shows the sensitivity and specificity of the test if the fifth quantile is used as the cutoff for IUGR (A) and after the elimination of cases under 20 weeks of gestation (B). There was increased sensitivity for the detection of SGA among cases over 19 weeks of gestation when a brain/liver weight ratio cutoff of 3 was used. Changing the cutoff for SGA to the fifth quantile had no significant effect on sensitivity or specificity.

A scatter plot of the birth weight quantiles of 179 fetuses against their ponderal index showed that most of the SGA fetuses had small ponderal indices but that many fetuses with small ponderal indices were not SGA (Fig. 4). When these data were examined by diagnostic class, Class 1 disease fetuses had significantly lower ponderal index than Class 2 fetuses $(P<0.0001$, ANOVA), but they were not significantly different from Class 3 fetuses (at $\alpha=0.05$, Hsu's MCB; Fig. 5).

The ponderal index was analyzed as a test for the detection of SGA. Table 4 shows the sensitivity and specificity of the test for each of 11 different cutoff points of ponderal index. The receiver operating curves for the brain/liver weight ratio and the ponderal index for the detection of SGA fetuses are shown in Fig. 6. The curve for ponderal index is considerably better than that for brain/liver weight ratio at every central level. There is no obvious optimum value for brain/liver weight ratio. The optimum value for ponderal index, taking the value from the apex of the curve, is a cutoff of 2.0. Some authors have used the tenth quantile of ponderal index at each gestational age as a cutoff value for normality $(2,14,18)$. Because

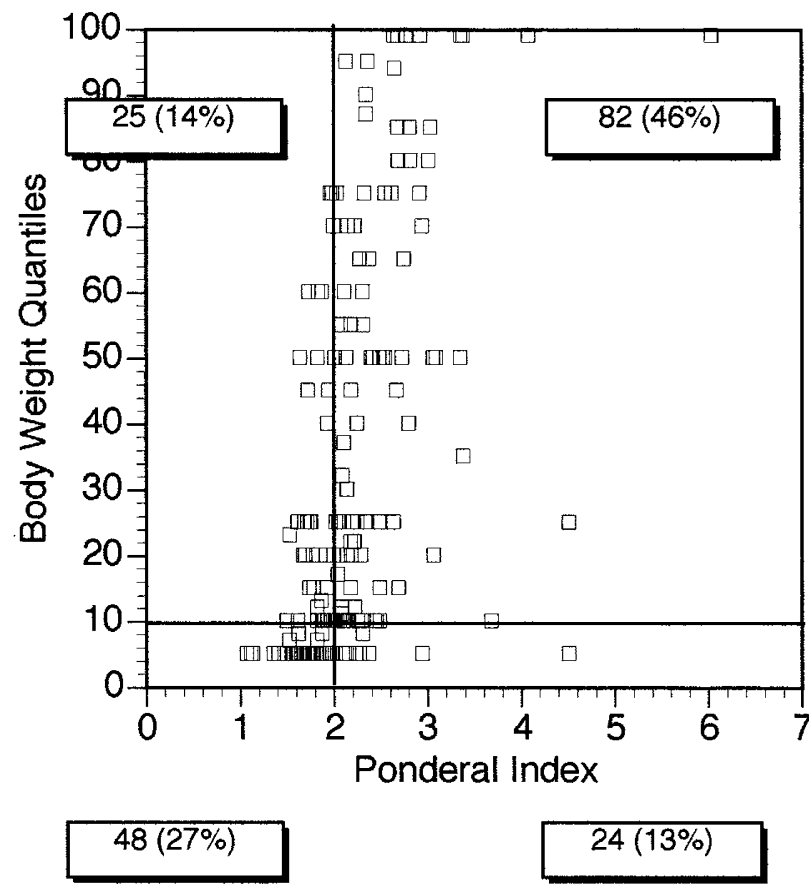

FIGURE 4. Fetal body weight quantile versus ponderal index. Reference lines drawn at ponderal index $=2$ and body weight quantile $=10$. Numbers and percentages in boxes are for the cases in each quadrant made by the reference lines. 


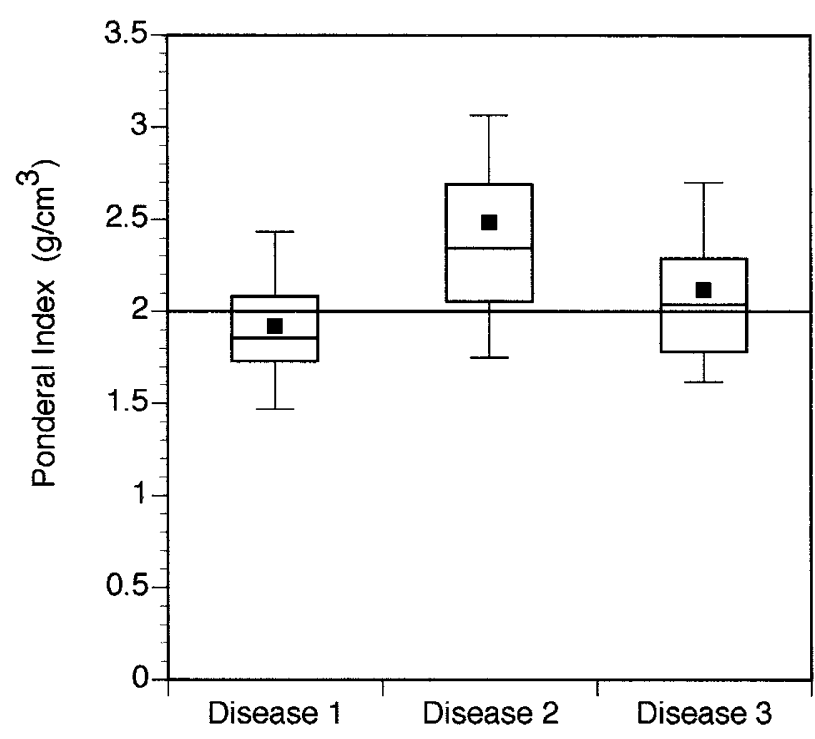

FIGURE 5. Box plots of each disease class distributed by ponderal index. Disease Class 1, likely associated with asymmetrical growth retardation, is different from Class 2 but not Class 3 by Hsu's MCB test at $\alpha=0.05$.

TABLE 3. Sensitivity and Specificity of Brain/Liver Weight Ratio for the Detection of SGA Fetus. A. SGA = Body Weight <5th. B. For Cases >19 Weeks Gestation.

\begin{tabular}{lll}
\hline & A & B \\
\hline Sensitivity & 54 & 83 \\
Specificity & 59 & 66 \\
\hline
\end{tabular}

TABLE 4. Sensitivity and Specificity of Ponderal Index for the Detection of SGA Fetus

\begin{tabular}{lllllll}
\hline PI cutoff: & 1.5 & 1.6 & 1.7 & 1.8 & 1.9 & 2.0 \\
Sensitivity & 8 & 17 & 31 & 40 & 56 & 67 \\
Specificity & 1 & 99 & 94 & 87 & 87 & 77 \\
& & & & & & \\
PI cutoff: & 2.1 & 2.2 & 2.3 & 2.4 & 2.5 & \\
Sensitivity & 76 & 86 & 92 & 93 & 99 & \\
Specificity & 67 & 55 & 47 & 37 & 34 & \\
\hline
\end{tabular}

the data in this study are from a population of stillborn infants, with a high proportion of SGA cases $(40 \%)$, the 10th quantile for each gestational age or age group was much too small to be a useful cutoff value. Rising values of ponderal index with gestational age were confirmed in our data.

Brain/liver weight ratio had poor correlation with ponderal index, $r=-0.17$ (Fig. 7).

The brain/liver weight ratio is not usually used as a simple test for IUGR. To more closely simulate the prosector's thought process at autopsy, the conditional sensitivity was calculated for brain/liver weight ratio with a cutoff of 3 for determining Disease Class 1, given that the fetus was in the bottom 10th quantile for body weight. That is, given that the fetus was small for gestational age and had a brain/liver weight ratio $>3$, how likely was it that the case would include maternal history or placen-

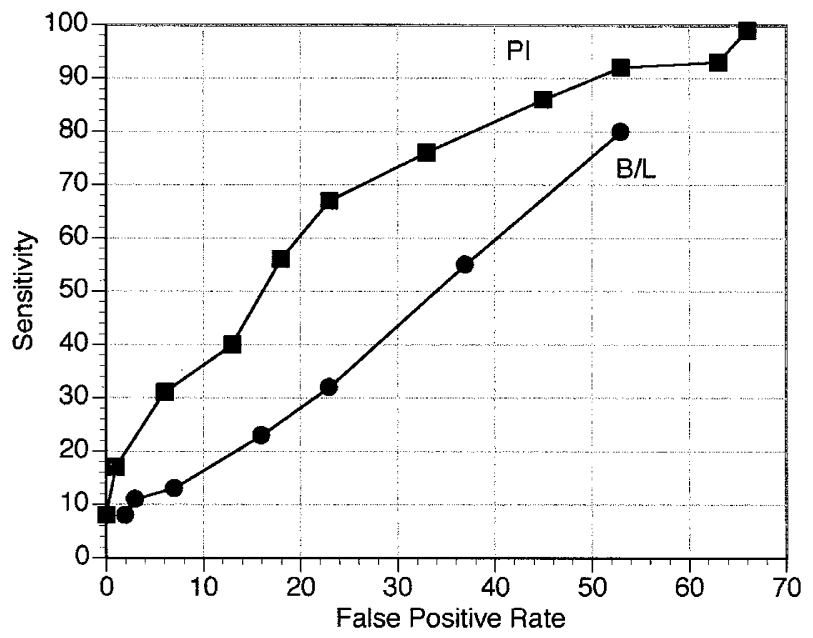

FIGURE 6. Receiver operating curve for detection of small-forgestational-age fetuses by fetal brain/liver weight ratio and by ponderal index.

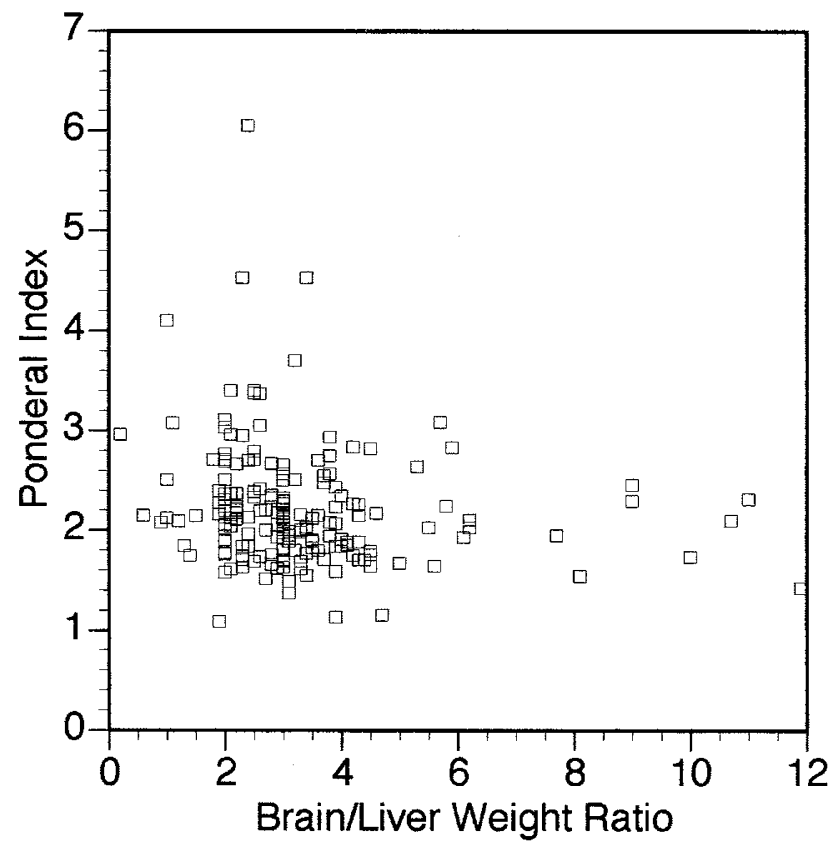

FIGURE 7. Fetal brain/liver weight ratio versus ponderal index. $r=$ -0.17 .

tal findings typical of asymmetrical growth retardation? This yielded a sensitivity of 1 and a specificity of 53\%; that is, all of the 10 SGA fetuses with a disease class of 1 had a brain/liver ratio $>3$, but most of the SGA fetuses had a disease class of 2 or 3 , regardless of brain/liver weight ratio value. Similar conditional sensitivity and specificity for ponderal index using a cutoff value of 2.0 were 10 and $61 \%$, respectively.

\section{DISCUSSION}

The concept of asymmetric- and symmetric-type growth retardation is accepted by many workers in 
perinatology and related fields and has been supported by experimental work with several different animal models (21-23). Furthermore, pathologists performing fetal autopsies find occasional cases with clear evidence of placental insufficiency (central or extensive parenchymal infarcts with maternal vascular changes), typical disproportionate fetal body habitus, and elevated brain/liver weight ratio. But some authors have shown evidence of an overall lack of clear separation between these IUGR categories. Kramer et al. (24) showed no evidence of bimodality in the distribution of 784 neonates that were classed as SGA and later showed that there was no tendency for growth-retarded infants to be disproportional with increasing gestational age (25). These workers suggested that asymmetrical growth retardation is a feature of severe growth retardation regardless of etiology. Crane et al. (26) studied ultrasonographic measurements in 33 growth-retarded infants and showed no difference in head circumference between infants classified as having either asymmetric or symmetric growth retardation. In response, some authors have commented that mixed forms of growth retardation are common and that the separation between types of growth retardation is a continuum and not always distinct (5, 27). Wigglesworth (5) stated that "the main difference between. . . [these categories of IUGR] is timing" but that "the pattern of growth impairment may indicate. . the most likely underlying cause."

This study analyzed data from 182 stillborn fetuses to attempt to determine the value of the brain/liver weight ratio for the detection of IUGR. The findings of this study are summarized below.

The ratio was insensitive and relatively nonspecific when used as a test for the detection of the SGA fetus. Sensitivity was increased when considering cases of at least 20 weeks of gestational age, and specificity was increased when the brain/liver weight ratio cutoff was increased over 3 .

The ratio had very poor correlation with ponderal index, a measure of thinness shown to be a relatively accurate measure of growth retardation and predictor of poor perinatal outcome $(2,14,15)$.

The ratio was statistically more likely to be elevated in cases with a maternal history compatible with asymmetrical growth retardation, but there was considerable overlap with other cases.

Ponderal index was considerably better than brain/liver ratio at detecting the SGA fetus, but this is perhaps expected in an index whose numerator is the fetal body weight.

When conditional sensitivity and specificity were measured, to more closely simulate the use of these indices at the time of autopsy, a brain/liver weight ratio $>3$ was very sensitive for the detection of a Disease Class 1 fetus among SGA fetuses but only had a specificity of $53 \%$. Similar conditional values for ponderal index showed very low sensitivity and relatively low specificity.

It is, of course, possible that some SGA fetuses in Disease Categories 2 and 3 with elevated brain/liver weight ratio actually did have asymmetric fetal growth retardation but were not detected by review of maternal history and examination of the placenta. Clinical history abstracts on the autopsy reports (and "complete" medical records, for that matter) may be incomplete; in particular, maternal cigarette smoking may have escaped detection. (Note that some researchers, however, have classified maternal cigarette smoking as a cause of symmetrical-type growth retardation [1]). In a meta-analysis examining 43 possible causal factors of IUGR, Kramer (28) found evidence that as many as one third of cases in developed countries might be attributable to cigarette smoking.

Prosectors of fetal autopsies must make use of all the information available to them. The fetal brain/ liver weight ratio and the ponderal index may be useful, but they may not be satisfactory indicators of IUGR by themselves, and they may also be inadequate for the detection of growth retardation etiology.

\section{REFERENCES}

1. Seeds J. Impaired fetal growth: definition and clinical diagnosis. Obstet Gynecol 1984;64:303-10.

2. Patterson R, Pouliot M. Neonatal morphometrics and perinatal outcome: who is growth retarded? Am J Obstet Gynecol 1987;157:691-3.

3. Adeniyi-Jones S. Intrauterine growth retardation. In: Spitzer AR, editor. Intensive care of the fetus and neonate. Philadelphia: Mosby; 1996. p. 137-48.

4. Wollmann H. Intrauterine growth restriction: definition and etiology. Horm Res 1998;49(2 Suppl):1-6.

5. Wigglesworth J. Perinatal pathology. 2nd ed. Philadelphia: Saunders; 1996. p. 7-12.

6. Balcazar H, Haas J. Classification schemes of small-forgestational age and type of intrauterine growth retardation and its implication to early neonatal mortality. Early Hum Dev 1990;24:219-30.

7. Lepercq J, Mahieu-Caputo D. Diagnosis and management of intrauterine growth retardation. Horm Res 1998;49(2 Suppl): 14-9.

8. Murphy J, Drumm J, Mulcahy R, Daly L. The effect of maternal cigarette smoking on fetal body weight and on growth of the fetal biparietal diameter. Br J Obstet Gynaecol 1980; $87: 462-6$.

9. Gruenwald P. Growth of the human fetus. II. Abnormal growth in twins and infants of mothers with diabetes, hypertension, or isoimmunization. Am J Obstet Gynecol 1966; 94:1120-32.

10. Chang TC, Robson SC, Boys RJ, Spencer JA. Prediction of the small for gestational age infant: which ultrasonic measurement is best? Obstet Gynecol 1992;80:1030-8.

11. Veille J, Cohen I. Middle cerebral artery blood flow in normal and growth-retarded fetuses. Am J Obstet Gynecol 1990;162: 391-6.

12. Deter R. Detection of fetal growth abnormalities. In: Deter R, Harrist R, Birnholz J, Hadlock F, editors. Quantitative obstetrical ultrasonography. New York: Wiley; 1986. p. 123-40. 
13. Benson C, Belville J, Lentini J, Saltzman D, Doubilet P. Intrauterine growth retardation: diagnosis based on multiple parameters-a prospective study. Radiology 1990;177:499-502.

14. Fay R, Dey P, Saadie C, Buhl J, Gebski V. Ponderal index: a better definition of the 'at risk' group with intrauterine growth problems than birth-weight for gestational age in term infants. Aust N Z J Obstet Gynaecol 1991;31:17-9.

15. Vintzileos A, Lodeiro J, Feinstein S, Campbell W, Weinbaum $\mathrm{P}$, Nochimson D. Value of fetal ponderal index in predicting growth retardation. Obstet Gynecol 1986;67:584-8.

16. Cole T, Henson G, Tremble J, Colley N. Birthweight for length: ponderal index, body mass index or Benn index? Ann Hum Biol 1997;24:289-98.

17. Lubchenco L, Hansman C, Boyd E. Intrauterine growth in length and head circumference as estimated from live births at gestational ages from 26-42 weeks. Pediatrics 1966;37:403-8.

18. Miller HC, Hassanein K. Diagnosis of impaired fetal growth in newborn infants. Pediatrics 1971;48:511-22.

19. Guihard-Costa A, Larroche J. Fetal biometry. Fetal Diagn Ther 1995;10:215-78.

20. Gruenwald P. Growth of the human fetus-I. Normal growth and its variations. Am J Obstet Gynecol 1966;94:1112-9.

21. Wigglesworth J. Fetal growth retardation. Animal model: uterine vessel ligation in the pregnant rat. Am J Pathol 1974; 77:347-50.
22. Harel S, Yavin E, Tomer A, Barak Y, Binderman I. Brain:body ratio and conceptional age in vascular-induced intrauterine growth retarded rabbits. Brain Dev 1985;7:575-9.

23. Flecknell P, Wootton R, John M, Royston J. Pathological features of intra-uterine growth retardation in the piglet: differential effects on organ weights. Diagn Histopathol 1981;4:295-8.

24. Kramer M, McLean F, Olivier M, Willis D, Usher R. Body proportionality and head and length "sparing" in growth retarded neonates: a critical reappraisal. Pediatrics 1989;84: 717-23.

25. Kramer M, Olivier M, McLean F, Dougherty G, Willis D, Usher R. Determinants of fetal growth and body proportionality. Pediatrics 1990;86:18-26.

26. Crane J, Kopta M. Comparative newborn anthropometric data in symmetric versus asymmetric intrauterine growth retardation. Am J Obstet Gynecol 1980;138:518-22.

27. Benson C, Doubilet P. Fetal measurements-normal and abnormal fetal growth. In: Rumack C, Wilson S, Charboneau J, editors. Diagnostic ultrasound. 2nd ed. Philadelphia: Mosby; 1998. p. 1013-31.

28. Kramer M. Determinants of low birth weight: methodological assessment and meta-analysis. Bull World Health Org 1987;65:663-737. 